\title{
Organ-Preserving Approach in Bladder Cancer: Assessment of the Current Situation
}

(D) Reha Girgin MD, (1) N. Aydın Mungan MD

Bülent Ecevit University Faculty of Medicine, Department of Urology, Zonguldak, Turkey

\begin{abstract}
Intravesical bacillus Calmette-Guerin (BCG) therapy is the gold standard treatment option in high-risk non-invasive bladder cancer. However, BCG is a very toxic agent. A significant proportion of patients have BCG intolerance after beginning intravesical treatment. Radical cystectomy is the recommended approach for patients with either BCG failure or BCG intolerance. Alternative intravesical salvage treatments are needed for patients who cannot tolerate radical cystectomy due to comorbidities or who refuse surgery.

In this review, current intravesical treatment alternatives to radical cystectomy in intravesical BCG failure are discussed with oncologic outcomes. Keywords: Bladder cancer, bacillus Calmette-Guérin failure, intravesical salvage therapy
\end{abstract}

\section{Introduction}

Bladder cancer is the seventh most common type of cancer among males and eleventh among both genders combined (1). In 2016 alone, 76,960 new cases were diagnosed and 16,390 cancer-related deaths were reported in the United States of America (2). Cancer staging and treatment planning can be achieved with a successful endoscopic resection and when necessary, metastatic evaluation. Although $70 \%$ of cases are non-muscle invasive superficial cancers (NMIBC) at the time of diagnosis, about $20 \%$ of these are grade Ta and T1 tumors including carcinoma in situ (CIS), which form a special group associated with high risk of cancer progression and relapse (3). Intravesical bacillus Calmette-Guérin (BCG) therapy is the primary treatment method for these high-risk NMIBCs (4). For non-metastatic muscle-invasive bladder cancer, radical cystectomy is considered the gold standard treatment option that provides the longest survival. With a goal of complete tumor elimination, grade 3-4 complication rate of $13 \%$ and mortality rate of $2.5-5.2 \%$ are considered acceptable (5).
Although NMIBC patients can be successfully treated with intravesical $B C G$, they exhibit relapse and progression rates of $80 \%$ and $40 \%$ respectively at 5 -year follow-up. As described in a 2000 study by the Southwestern Oncological Group (SWOG), the intravesical BCG regimen is administered as a 6-week induction course followed by 3 years of maintenance therapy if there are no signs of tumor in follow-up endoscopic examinations (6). However, in the presence of persistent or recurrent NMIBC, a substantial proportion of patients may benefit from an additional 6-week induction course with or without interferon (IFN) alpha (3). Findings indicating BCG resistance during therapy include high-grade NMIBC in the first cystoscopic examination, CIS in the first or second cystoscopic examination, and high-grade cancer in follow-up cystoscopic examinations during or after completing the intravesical BCG protocol (7). The occurrence of adverse effects that prevent continuation of treatment is referred to as BCG intolerance $(7,8)$. NMIBC patients exhibiting BCG resistance or intolerance are candidates for radical cystectomy (9). However, the various comorbidities in elderly patients and the reluctance of younger patients to risk undergoing such a 
procedure have led to a search for local therapeutic alternatives to radical cystectomy (5).

\section{Mitomycin C}

Thiotepa, adriamycin, and mitomycin C (MMC) were initially the preferred first-line agents for patients who were sensitive to BCG but could not undergo BCG therapy (9). Today, only MMC still holds this position (10). MMC has an important place among chemotherapeutic agents. It is an alkylating agent that disrupts DNA synthesis (4). It is used perioperatively in NMIBC treatment due to its ability to block tumor seeding in particular (4). However, maintenance therapy does not yield satisfactory outcomes when BCG fails (10). To that end, it seems that advances in intravesical drug administration techniques are starting to provide favorable outcomes. Chemohyperthermia $(\mathrm{CHT})$ and electromotive drug administration (EMDA) are two methods developed to achieve this aim. In $\mathrm{CHT}$, a specialized urethral catheter is used to deliver radiofrequency waves inside the bladder, thus raising the temperature. This increases cell permeability to MMC and promotes apoptosis by inducing stress in tumor cells (11). After using this technique in 111 patients with failed BCG, Nativ et al. (12) reported recurrencefree rates of $85 \%$ at 1 year and $56 \%$ at 2 years. In another study including 51 patients with failed BCG from 15 centers in Europe, Witjes et al. (13) reported complete response rates of $92 \%$ initially and $50 \%$ after 2 years. Although $\mathrm{CHT}$ is recognized by many authorities, it is still in the Food and Drug Administration (FDA) review process. EMDA aims to create an electromagnetic field to increase bladder surface epithelial cell permeability to MMC (14). Like CHT, EMDA was designed to increase the efficacy of MMC in moderate and high-risk NMIBC, but was unable to provide satisfactory results when applied after resistance to BCG. Sockett et al. (15) reported $31 \%$ relapse at 15 months in 13 patients with failed BCG who were given MMC by EMDA.

\section{Gemcitabine}

Gemcitabine (GC) is a nucleotide antimetabolite that disrupts DNA synthesis in tumor cells by inhibiting ribonucleotide reductase and cystine diaminase (4). In a randomized controlled study, Addeo et al. (16) determined that GC was more effective and less toxic than MMC. In their phase 2 trial, Skinner et al. (17) reported a recurrence-free rate of $28 \%$ after 1 year of treatment with intravesical GC. Prasanna et al. (18) also reported in their study that intravesical GC provided a similar disease-free survival to intravesical BCG and caused less toxicity. Although intravesical BCG is currently the gold standard treatment for high-risk NMIBC, GC may be recommended as an alternative first-line intravesical therapy for BCG-resistant patients not suitable for cystectomy and patients who cannot tolerate the toxicity of BCG (18).

\section{Valrubicin}

Valrubicin is a synthetic anthracycline analogue that exerts a toxic effect by penetrating nucleic acid sequences and arresting the cell cycle (19). Steinberg et al. (19), who comprise the valrubicin study group, reported a complete response rate of $21 \%$ and disease-free rate of $8 \%$ at the end of 18 months follow-up in 90 patients with BCG-refractory CIS. As a result of this study, intravesical valrubicin therapy is the only chemotherapeutic agent approved by the FDA for BCGrefractory CIS (20).

\section{Taxanes (Docetaxel and Paclitaxel)}

Agents in the taxane group act by disrupting microtubule function and halting cell division at M-phase (21). Preclinical studies have shown that taxane chemotherapeutics are highly effective on bladder cancer cells (22). Laudano et al. (22) conducted a phase 1 trial in which intravesical docetaxel (DTX) was administered to 18 patients who did not respond to intravesical BCG, and reported a complete response rate of $22 \%$, partial response rate of $17 \%$, and non-response rate of $61 \%$ during a mean follow-up of 48 months (22). In another study by Barlow et al. (23) 54 non-responders to BCG where administered intravesical DTX, and recurrence-free rates at 1 and 3 years were $40 \%$ and $25 \%$, respectively.

Intravesical agents must remain in the bladder for 2 hours to achieve maximum efficacy (24). However, due to reasons such as bladder irritability or low bladder capacity, this waiting period is often unachievable (24). Since paclitaxel (PTX) was first introduced in 1967, many carrier agents have been investigated to increase the efficacy of taxanes due to their lipophilic properties and low cell penetration (4). In the phase 1 study by McKiernan et al. (25) using PTX bound to intravesical nanoparticle albumin (NPA), the complete response rate at 6 weeks was $28 \%$ and only grade 1 toxicity occurred in 10 of the 18 patients. In a subsequent phase 2 study, the complete response rate was $35.7 \%$ and toxicity rate was $32.1 \%$ at 1 year (26). Robins et al. (27) recently reported a disease-free rate of $18 \%$ and a cancer-specific survival rate of $9 \%$ at the end of 41 months follow-up in patients treated with NPA-bound PTX after BCG failure.

\section{Interferon}

IFN is a cytokine with immunomodulatory, antiproliferative, and antiviral properties (28). Earlier research established that IFN monotherapy had no utility in the treatment of NMIBC (28). The first of these studies was a 1990 prospective randomized study by Glashan (29) including 87 patients who were treated with either low-dose (10 million $U$ ) or high-dose (100 million U) intravesical IFN monotherapy and followed for 12 months. After 1 year, complete response rate was $43 \%$ in the high-dose arm and 5\% in the low-dose arm (29). The most common side effects were influenza-like symptoms, which occurred in $17 \%$ of patients in the high-dose arm and $8 \%$ of those in the low-dose arm (29). However, in 1995, Hudson and Ratliff (30) conducted a prospective study with 12 patients who were treated with intravesical IFN (100 million $\mathrm{U}$ ) after non-response to previous BCG treatment, and they reported a complete response rate of $8 \%$ at the end of 24 months. In a multi-center randomized phase 2 trial of intravesical BCG and IFN combined therapy conducted by Joudi et al. (31) a cancer-free survival rate of $13 \%$ was reported at the end of 24 months follow-up. In another prospective study including 50 patients treated 
with a combination of BCG and IFN, Bazarbashi et al. (32) reported that $62 \%$ of the patients were recurrence-free after a median follow-up of 55.8 months. Eighteen percent of the patients developed grade 3 dysuria and 14\% developed grade 3 frequency (32).

\section{Mycobacterial Cell Wall Extracts}

Intravesical BCG, currently the gold standard treatment for high-risk NMIBC, seems to stimulate an inflammatory response in target cells (33). Research on mycobacteria first started in 1970 with animal studies, and the first study regarding its successful intravesical use in humans was published by Morales et al. (34) in 1976. Although treatment with BCG obtained from live attenuated mycobacteria is undeniably effective, it also gives rise to local and systemic adverse effects (33). This has led to a search for more effective and less toxic agents (33). Mycobacterium phlei cell wall and mycobacterial cell wall-nucleic acid complex (MCNA) were tested in the treatment of NMIBC in 1996 and 1997, respectively $(35,36)$. Morales et al. $(37,38)$ first published initial results with the purportedly immunomodulatory and cytotoxic MCNA in 2001, and in a recent phase 3 trial of MCNA for BCG-resistant patients published in 2015, they reported complete response rates of $22 \%$ and $19 \%$ at 1 year and 2 years, respectively (36). However, MCNA failed to gain FDA approval in 2016 (39).

\section{Targeted Therapies}

Bladder cancer is one of the most immunogenic cancers, with high rates of somatic mutation (40). One of the unique features of the bladder is that it forms a defense against microorganisms without eliciting an immune response (41). This makes it rather difficult to generate an anti-tumor response against bladder tumors (41). Bladder tumor cells evade the immune system using immune checkpoints that block T-lymphocyte defense. Programmed death-1 (PD-1), programmed death ligand-1 (PDL1), and cytotoxic T-lymphocyte-associated protein-4 are the most extensively studied checkpoints (42). Research has been based on the premise that immune checkpoint blockade would shrink the tumor cells. The search for less toxic second-line agents for metastatic bladder cancer patients who cannot be treated with cisplatin (MDP)-based chemotherapeutic agents resulted in the discovery of targeted agents, some of which have been approved by the FDA (43). Current research is focusing on targeted agents to be used in NMIBC when BCG fails (43). Phase 2 studies of anti-PD-1/PD-L1 seem promising for patients with intravesical BCG-refractory $\operatorname{NMIBC}(44,45)$. Intravesical use of targeted agents to reduce systemic side effects is an important and necessary area of research (46). VB4845 is an immunomodulatory recombinant protein bound to Pseudomonas exotoxin that can be administered intravesically (9). This agent causes apoptosis of tumor cells, but a complete response rate of only $16 \%$ at 12 months was reported in a phase 2 trial (9).

\section{Oncoviral Agents}

The use of viral agents for tumor control is an area of intensive study in current medical practice. Inducing tumor cell lysis (oncolysis) via oncolytic viral agents can be achieved by direct stimulation of infected cells, by indirect stimulation of noninfected cells, or by stimulation of the immune system (47). In a phase 1 trial by Burke et al. (48) evaluating intravesical administration of CG0070 (adenoviral agents expressing granulocyte-macrophage colony-stimulating factor ([GM-CSF]) in 3 sessions over 28 days or in 6 weekly sessions, the response rate was $48.6 \%$ and the complete response rate was $61.5 \%$ at even the lowest doses. A single-arm, multi-center phase 3 trial evaluating the safety and efficacy of GC0070 in NMIBC patients who have previous BCG failure and refuse cystectomy is still in progress (47).

\section{Photodynamic Treatment Approaches}

Photodynamic therapy (PDT) is a method in which photosensitized agents are activated by specific wavelengths of light to cause apoptosis and necrosis of tumor cells (49). Dating back to the early $20^{\text {th }}$ century, PDT has been used for tumor treatment in many fields (49). In 1976, Kelly and Snell (50) reported the first data on the use of PDT in bladder cancer. However, the degree of systemic toxicity necessitated the development of new agents. The first data on the use of 5-aminolevulinic acid (5-ALA) were reported by Kriegmair et al. (51) in 1996. Intravenous administration of 5-ALA in PDT resulted in a complete response rate of $31 \%$ at 1 year, and $19 \%$ of the patients experienced bladder spasms as an adverse effect (49). In a study by Lee et al. (52) including 34 patients, tumor-free survival rate was $90 \%$ at 12 months and $60 \%$ at 30 months. Berger et al. (53) reported a complete response rate of $40 \%$ at 1 year in patients treated with intravesical 5-ALA. When hexaminolevulinic acid was used for the same purpose, the complete response rate at 1 year was 12\% (54). Although low response rates prevent the widespread use of PDT, it may become relevant as an alternative therapy in the future.

\section{Combined Therapies}

Because different chemotherapeutic agents have different mechanisms of action, combined therapies are utilized to create synergistic effects for cancer treatment (4). This known property of chemotherapeutics in cancer treatment has been investigated in salvage intravesical applications in patients with failed BCG (4).

In a study evaluating intravesical administration of an adriamycin and MMC combination, Fukui et al. (55) reported that 13 of $30 \mathrm{CIS}$ patients were completely tumor-free at the end of 23 months follow-up, but the high rate of local toxicity (70\%) compelled the researchers to seek new combinations.

In a retrospective study by Cockerill et al. (56) including 27 patients, 37\% were recurrence-free at 22 months follow-up while recurrence was detected at a mean of 15 months in the other $63 \%$, and 1 patient (3.7\%) showed progression during treatment. In another retrospective multi-center study in which 47 patients with failed BCG received intravesical combined GC/MMC, Lightfoot et al. (57) reported a recurrence-free rate of $48 \%$ at 1 year and $38 \%$ at 2 years. The first known data on treatment with intravesical GC/DTX in patients with failed BCG were published by Steinberg et al. (58) who reported a 
recurrence-free rate of $54 \%$ at 1 year and $34 \%$ at 2 years, and a complete response rate of $66 \%$. Milbar et al. (59) retrospectively analyzed the data of 33 patients who received intravesical GC/ DTX therapy and reported recurrence-free rates of $56 \%$ and $42 \%$ at 1 and 2 years, respectively. Only $2(3 \%)$ patients could not tolerate the treatment (59).

Chen et al. (60) compared the success rates of BCG with those of an intravesical MMC, doxorubicin, and MDP protocol, and reported comparable recurrence rates at 5 years $(37.9 \%$ vs $33.9 \%$ ). With a $5.8 \%$ major adverse event profile, intravesical MDP seems superior to intravesical BCG, which had a $15 \%$ major adverse event profile (60). Despite these data, further research is needed on intravesical MDP therapy in patients resistant to intravesical BCG.

In a study of 54 patients with BCG failure, Steinberg et al. (61) added intravesical interleukin-2 and subcutaneous GM-CSF to intravesical BCG and IFN therapy, and reported success rates of $55 \%$ and $53 \%$ at 1 and 2 years, respectively. Treatment intolerance was observed in $6 \%$ of the patients (61).

\section{Conclusion}

Intravesical BCG has been used in high-risk NMIBC for nearly half a century (9). Nevertheless, radical cystectomy is still recommended in patients with resistance to or intolerance of intravesical BCG therapy. However, the FDA has also acknowledged the need for intravesical salvage therapy for patients who are ineligible for or refuse a complicated surgery like cystectomy, and research is being supported to accelerate the discovery of new agents for this patient group (3). All of the treatments described above appear to offer some degree of success, but most of the studies are focused on small and heterogeneous patient groups with short follow-up periods.

Consequently, therapies that are potentially useful for high-risk NMIBC patients with limited options should be reevaluated with appropriate endpoints.

\section{Ethics}

Peer-review: Externally peer-reviewed.

\section{Authorship Contributions}

Concept: N.A.M., R.G. Design: N.A.M., Data Collection or Processing: R.G., Analysis or Interpretation: N.A.M., R.G., Literature Search: R.G., Writing: R.G.

Conflict of Interest: No conflict of interest was declared by the authors.

Financial Disclosure: The authors declared that this study received no financial support.

\section{References}

1. Ferlay J, Soerjomataram I, Ervik M, et al. GLOBOCAN 2012 v1.0: Estimated cancer incidence, mortality and prevalence worldwide in 2012. 2013. 2015. http://globocan.iarc.fr, accessed on day/month/year

2. Siegel RL, Miller KD, Jemal A. Cancer statistics, 2016. CA Cancer J Clin 2016;66:7-30.

3. Edward M. Messing. Bladder Sparing Therapy for BCG Failures - I Intravesical Immunotherapy. Bladder Cancer 2017;3:313-314.

4. Ahn JJ, Ghandour RA, McKiernan JM. New agents for bacillus Calmette-Guerin-refractory nonmuscle invasive bladder cancer. Curr Opin Urol 2014;24:540-545.
5. Lamm DL, Blumenstein BA, Crissman JD, et al. Maintenance bacillus Calmette-Guerin immunotherapy for recurrent TA, T1 and carcinoma in situ transitional cell carcinoma of the bladder: a randomized Southwest Oncology Group study. J Urol 2000;163:1124-1129.

6. Mostafid AH, Palou Redorta J, Sylvester R, Witjes JA. Therapeutic options in high-risk non-muscle-invasive bladder cancer during the current worldwide shortage of bacille Calmette-Guerin. Eur Urol 2015;67:359-360.

7. Babjuk $M$, Böhle $A$, Burger $M$, et al. EAU guidelines on non-muscleinvasive urothelial carcinoma of the bladder: update 2016. Eur Urol 2017;71:447-461.

8. Alhogbani MM, Picard JA, Fassi-Fehri MH, et al. Prognostic impact of Bacillus Calmette-Guérin interruption at the time of induction and consolidation. Urol Ann 2017;9:315-320.

9. Packiam VT, Johnson SC, Steinberg GD. Non-Muscle-Invasive Bladder Cancer: Intravesical Treatments Beyond Bacille Calmette-Guerin. Cancer 2017;123:390-400.

10. Malmström PU, Wijkström $\mathrm{H}$, Lundholm $\mathrm{C}$, et al. 5-year followup of a randomized prospective study comparing mitomycin $\mathrm{C}$ and bacillus Calmette-Guerin in patients with superficial bladder carcinoma. Swedish Norwegian Bladder Cancer Study Group. J Urol 1999;161:1124-1127.

11. Lammers RJ, Witjes JA, Inman BA, et al. The role of a combined regimen with intravesical chemotherapy and hyperthermia in the management of nonmuscle-invasive bladder cancer: a systematic review. Eur Urol 2011;60:81-93.

12. Nativ O, Witjes JA, Hendricksen K, et al. Combined thermochemotherapy for recurrent bladder cancer after bacillus CalmetteGuerin. J Urol 2009;182:1313-1317.

13. Witjes JA, Hendricksen K, Gofrit O, et al. Intravesical hyperthermia and mitomycin- $C$ for carcinoma in situ of the urinary bladder: experience of the European Synergo working party. World J Urol 2009;27:319-324.

14. Zargar H, Aning J, Ischia J, et al. Optimizing intravesical mitomycin C therapy in nonmuscle-invasive bladder cancer. Nat Rev Urol 2014;11:220-230.

15. Sockett LJ, Borwell J, Symes A, et al. Electro-motive drug administration (EMDA) of intravesical mitomycin-C in patients with high-risk noninvasive bladder cancer and failure of BCG immunotherapy. BJU Int 2008;101(Suppl 5):50.

16. Addeo R, Caraglia M, Bellini S, et al. Randomized phase III trial on gemcitabine versus mytomicin in recurrent superficial bladder cancer: evaluation of efficacy and tolerance. J Clin Oncol 2010;28:543-548.

17. Skinner EC, Goldman B, Sakr WA, et al. SWOG S0353: Phase II trial of intravesical gemcitabine in patients with nonmuscle invasive bladder cancer and recurrence after 2 prior courses of intra vesical bacillus Calmette-Guérin. J Urol 2013;190:1200-1204.

18. Prasanna T, Craft P, Balasingam G, et al. Intravesical Gemcitabine versus Intravesical Bacillus Calmette-Guérin for the Treatment of Non-Muscle Invasive Bladder Cancer: An Evaluation of Efficacy and Toxicity. Front Oncol 2017;7:260.

19. Steinberg G, Bahnson R, Brosman S, et al. Efficacy and safety of valrubicin for the treatment of bacillus Calmette-Guerin refractory carcinoma in situ of the bladder. The Valrubicin Study Group. J Urol 2000;163:761-767.

20. Porten SP, Leapman MS, Greene KL. Intravesical chemotherapy in non-muscle-invasive bladder cancer. Indian J Urol 2015;31:297-303.

21. Calabro F, Sternberg CN. Localized and locally advanced bladder cancer. Curr Treat Options Oncol 2002;3:413-428.

22. Laudano MA, Barlow LJ, Murphy AM, et al. Long-term clinical outcomes of a phase I trial of intravesical docetaxel in the management of non-muscle-invasive bladder cancer refractory to standard intravesical therapy. Urology 2010;75:134-137.

23. Barlow LJ, McKiernan JM, Benson MC. Long-term survival outcomes with intravesical docetaxel for recurrent nonmuscle invasive bladder cancer after previous bacillus Calmette-Guérin therapy. J Urol 2013; 189:834-839. 
24. Tamura K, Kikuchi E, Konno T, et al. Therapeutic effect of intravesical administration of paclitaxel solubilized with poly(2methacryloyloxyethyl phosphorylcholine-co-n-butyl methacrylate) in an orthotopic bladder cancer model. BMC Cancer 2015;15:317.

25. McKiernan JM, Barlow LJ, Laudano MA, et al. A phase I trial of intravesical nanoparticle albumin-bound paclitaxel in the treatment of bacillus Calmette-Guerin refractory nonmuscle invasive bladder cancer. J Urol 2011;186:448-451.

26. McKiernan JM, Holder DD, Ghandour RA. Phase II trial of intravesical nanoparticle albumin bound paclitaxel for the treatment of nonmuscle invasive urothelial carcinoma of the bladder after bacillus Calmette-Guérin treatment failure. J Urol 2014;192:1633-1638.

27. Robins DJ, Sui W, Matulay JT, et al. Long-Term Survival Outcomes with Intravesical Nanoparticle Albumin-Bound Paclitaxel for Recurrent Nonmuscle Invasive Bladder Cancer after Previous Bacillus CalmetteGuérin Therapy. Urology. 2017;103:149-153.

28. Belldegrun AS, Franklin JR, O'Donnell MA, et al. Superficial bladder cancer: the role of interferon-alpha. J Urol 1998;159:1793-1801.

29. Glashan RW. A randomized controlled study of intravesical alpha2b-interferon in carcinoma in situ of the bladder. J Urol 1990;144:658-661.

30. Hudson MA, Ratliff TL. Failure of intravesical interferon-alfa-2b for the treatment of patients with superficial bladder cancer previously failing intravesical BCG therapy. Urol Oncol 1995;1:115-118.

31. Joudi FN, Smith BJ, O'Donnell MA, et al. Final results from a national multicenter phase II trial of combination bacillus Calmette-Guerin plus interferon $\mathrm{a}-2 \mathrm{~B}$ for reducing recurrence of superficial bladder cancer. Urol Oncol 2006;24:344-348.

32. Bazarbashi S, Soudy $H$, Abdelsalam $M$, et al. Co-administration of intravesical bacillus Calmette-Guérin and interferon $\alpha-2 B$ as first line in treating superficial transitional cell carcinoma of the urinary bladder. BJU Int 2011;108:1115-1118.

33. Ertürk $Z$, Büber $E$, Özen $H$, et al. Mycobacterium Brumae Extract Fractions with Potential Immunotherapeutic Activity for Bladder Cancer. Bull Urooncol 2017;16 70-76.

34. Morales A, Eidinger D, Bruce AW. Intracavitary Bacillus CalmetteGuerin in the treatment of superficial bladder tumors. J Urol 1976;116:180-183.

35. Chin JL, Kadhim SA, Batislam E, et al. Mycobacterium cell wall: an alternative to intravesical bacillus Calmette Guerin (BCG) therapy in orthotopic murine bladder cancer. J Urol 1996;156:1189-1193.

36. Filion MC, Lepicier P, Morales A, Philips NC. Mycobacterium phlei cell wall complex directly induces apoptosis in human bladder cancer cells. Br J Cancer 1999;79:229-235.

37. Morales A, Chin JL, Ramsey EW. Mycobacterial cell wall extract for treatment of carcinoma in situ of the bladder. J Urol 2001; 166:1633-1637.

38. Morales A, Herr H, Steinberg G, et al. Efficacy and safety of MCNA in patients with nonmuscle invasive bladder cancer at high risk for recurrence and progression after failed treatment with bacillus Calmette-Guerin. J Urol 2015;193:1135-1143.

39. Packiam VT, Johnson SC, Steinberg GD. Non-muscle-invasive bladder cancer: Intravesical treatments beyond Bacille Calmette-Guérin. Cancer 2017;123:390-400.

40. Kandoth C, McLellan MD, Vandin F, et al. Mutational landscape and significance across 12 major cancer types. Nature 2013;502:333-339.

41. Smith SG, Zaharoff DA. Future directions in bladder cancer immunotherapy: towards adaptive immunity. Immunotherapy 2016;8:351-365.

42. Baksh K, Weber J. Immune checkpoint protein inhibition for cancer: preclinical justification for CTLA-4 and PD-1 blockade and new combinations. Semin Oncol 2015;42:363-377.

43. Petrylak DP. Immunotherapy: The Wave of Future in Bladder Cancer? Clin Genitourin Cancer 2017;15:3-17.
44. Vandeveer AJ, Fallon JK, Tighe R, et al. Systemic Immunotherapy of Non-Muscle Invasive Mouse Bladder Cancer with Avelumab, an Anti-PD-L1 Immune Checkpoint Inhibitor. Cancer Immunol Res 2016;4:452-462.

45. Kamat AM, Belmunt J, Choueiri TK, et al. KEYNOTE-057:phase 2 study of pembrolizumab for patient (pts) with Bacillus Calmette Guaren (BCG)-unresponsive, high-risk non-muscle-invasive bladder cancer (NMIBC) [abstract]. J Clin Oncol 2016;34(Suppl):4576.

46. Smith SG, Zaharoff DA. Future directions in bladder cancer immunotherapy: towards adaptive immunity. Immunotherapy 2016;8:351-365.

47. Hamid O, Hoffner B, Gasal E, et al. Oncolytic immunotherapy: unlocking the potential of viruses to help target cancer. Cancer Immunol Immunother 2017;66:1249-1264.

48. Burke JM, Lamm DL, Meng MV, et al. A first in human phase 1 study of CG0070, a GM-CSF expressing oncolytic adenovirus, for the treatment of nonmuscle invasive bladder cancer. J Urol 2012; 188:2391-2397.

49. Inoue K. 5-Aminolevulinic acid-mediated photodynamic therapy for bladder cancer. Int J Urol 2017;24:97-101.

50. Kelly JF, Snell ME. Hematoporphyrin derivative: a possible aid in the diagnosis and therapy of carcinoma of the bladder. J Urol 1976;115:150-151.

51. Kriegmair M, Baumgartner R, Lumper W, et al. Early clinical experience with 5-aminolevulinic acid for the photodynamic therapy of superŞcial bladder cancer. Br J Urol 1996;77:667-671.

52. Lee JY, Diaz RR, Cho KS, et al. Efficacy and safety of photodynamic therapy for recurrent, high grade nonmuscle invasive bladder cancer refractory or intolerant to bacille Calmette-Guerin immunotherapy. J Urol 2013;190:1192-1199.

53. Berger AP, Steiner H, Stenzl A, et al. Photodynamic therapy with intravesical instillation of 5 -aminolevulinic acid for patients with recurrent superficial bladder cancer: a single-center study. Urology 2003;61:338-341.

54. Bader MJ, Stepp H, Beyer W, et al. Photodynamic therapy of bladder cancer - a phase I study using hexaminolevulinate (HAL). Urol Oncol 2013;31:1178-1183.

55. Fukui I, Sekine H, Kihara K, et al. Intravesical combination chemotherapy with mitomycin $\mathrm{C}$ and doxorubicin for carcinoma in situ of the bladder. J Urol 1989;141:531-534.

56. Cockerill PA, Knoedler JJ, Frank I, et al. Intravesical gemci tabine in combination with mitomycin $C$ as salvage treatment in recurrent non-muscle-invasive bladder cancer. BJU Int 2016;117:456-462.

57. Lightfoot AJ, Breyer BN, Rosevear HM, et al. Multi-institutional analysis of sequential intravesical gemcitabine and mitomycin $C$ chemotherapy for non-muscle invasive bladder cancer. Urol Oncol 2014;32:35.

58. Steinberg RL, Thomas LJ, O'Donnell MA, et al. Sequential Intravesical Gemcitabine and Docetaxel fort the Salvage Treament of NonMuscle Invasive Bladder Cancer. BI Cancer 2016;1:65-72.

59. Milbar N, Kates M, Chappidi MR, et al. Oncological Outcomes of Sequential Intravesical Gemcitabine and Docetaxel in Patients with Non-Muscle Invasive Bladder Cancer. Bladder Cancer 2017;3:293-303.

60. Chen $\mathrm{CH}$, Yang $\mathrm{H}$, Shun $\mathrm{CT}$, et al. A cocktail regimen of intravesical mitomycin-C, doxorubicin, and cisplatin (MDP) for non-muscleinvasive bladder cancer. Urol Oncol 2012;30:421-427.

61. Steinberg RL, Nepple KG, Velaer KN, et al. Quadruple immunotherapy of Bacillus Calmette-Guérin, interferon, interleukin-2, and granulocytemacrophage colony-stimulating factor as salvage therapy for nonmuscle-invasive bladder cancer. Urol Oncol 2017;35:670. 